\title{
¿Cómo lograr la excelencia sanitaria en la atención del paciente con enfermedad renal crónica? Desarrollo de vías clínicas para los estadios iniciales de la enfermedad
}

\author{
Eduardo Navarro Jiménez ${ }^{(D)}{ }^{1} \square$, Carmen Laborde Cárdenas ${ }^{(D)}{ }^{1}$, Gustavo \\ Aroca Martínez (D) ${ }^{1,2}$, Victoria Nicholls De La Hoz ${ }^{(D)}{ }^{1}$, Camila Hernández \\ Berrios ${ }^{(D 1}{ }^{1}$, Bryan Olivero Pacheco ${ }^{(D)}{ }^{1}$ y Luis Castillo Parodi (D) 2,3 \\ ${ }^{1}$ Facultad de Ciencias de la Salud, Universidad Simón Bolívar, Barranquilla, Colombia. \\ ${ }^{2}$ Centro de Investigación, Clínica de la Costa, Barranquilla, Colombia. \\ ${ }^{3}$ Departamento de Medicina Interna, Universidad Cooperativa de la Costa, Santa Marta, Colombia.
}

Recibido:

08/Abr/2020

Aceptado:

02/Jun/2021

Publicado:

18/Ago/2021
Cómo citar: Navarro Jiménez E, Laborde Cárdenas C, Aroca Martínez G, Nicholls De La Hoz V, Hernández Berrios C, Olivero Pacheco B. ¿Cómo lograr la excelencia sanitaria en la atención del paciente con enfermedad renal crónica? Desarrollo de vías clínicas para los estadios iniciales de la enfermedad. Rev. Colomb. Nefrol. 2021 8(3), e411. https:/doi.org/10.22265/acnef.8.3.411

$\square$ Correspondencia: Carrera 59\#59. Barranquilla, Colombia. enavarro27@unisimonbolivar.edu.co

Las vías clínicas (VC) son instrumentos desarrollados para planificar y coordinar la secuencia de los procedimientos clínicos y administrativos necesarios para conseguir la máxima calidad asistencial. La rápida difusión de las VC en Estados Unidos explica que más del $60 \%$ de los hospitales usen estos documentos para sus procesos más frecuentes, lo que contrasta con su escasa implementación en Colombia y países de Latinoamérica. Las VC se ajustan según los cambios que representa el enfoque en procesos y se orientan a optimizar una mejor atención al paciente.

Palabras clave: vías clínicas, garantía de la calidad de atención de salud, calidad de la atención de salud, enfermedades renales. 


\section{How to achieve health excellence in the care of patients with chronic kidney disease? Development of clinical pathways for the initial stages of the disease}

\section{Abstract}

The clinical pathways are instruments developed to plan and coordinate the sequence of clinical and administrative procedures necessary to achieve the highest quality of care. Its rapid spread in the USA. USA explains that more than $60 \%$ of hospitals use clinical pathways for their most frequent processes. This contrasts with its poor implementation in Colombia and the countries of the Region of the Americas. The Clinical Pathways adjust with the change represented by the focus on processes, aimed at optimizing better patient care.

Keywords: critical pathways, health care quality assurance, quality of health care, kidney diseases.

\section{Introducción}

Las vías clínicas (VC) se definen como una herramienta de planificación o diseño de la calidad cuya implementación debe monitorizarse con los indicadores adecuados para determinar si se están aplicando adecuadamente y si con su implementación se están consiguiendo los resultados esperados en atención sanitaria [1]. Las VC se alinean plenamente con el cambio que representa el enfoque de los procesos orientados a optimizar los resultados, en este caso una mejor atención al paciente.

Las VC son un apoyo que, con base en un enfoque de optimización de simulación, permiten identificar el ajuste y la alineación adecuados de los recursos para lograr un mejor rendimiento que sea beneficioso tanto para los pacientes como para el centro de atención médica [2]. Estos documentos garantizan la coordinación, la eficiencia, la calidad y la seguridad en entornos de atención en salud caóticos [3], por lo que terminan siendo planes asistenciales estandarizados que se aplican a un grupo homogéneo de pacientes con un curso clínico predecible en un periodo de tiempo específico [4]. En la presente revisión se describe la importancia de la implementación y la aplicabilidad de las VC en entornos clínicos de enfermedad renal crónica (ERC) [5]. 


\section{Selección de procesos para el desarrollo de una VC}

Las VC facilitan la gestión de los procesos gracias a su utilidad para definirlos, evaluarlos, mejorarlos y homogenizarlos. Existen criterios para determinar qué procesos se deben sistematizar mediante una VC, algunos de estos son:

- Proceso o procedimiento con un curso clínico predecible

- Proceso prevalente o recurrente

- Estancias hospitalarias prolongadas

- Costos elevados

- Variabilidad no justificada

- Participación de varias especialidades

- Posibilidades de mejora

- Reclamaciones y/o quejas reiteradas

- Referencias de estándares elevados

Asimismo, las VC se pueden aplicar a diversos estadios o fases de un proceso asistencial. Esto se hace en función de la importancia que puede tener el hecho de sistematizar cada una de dichas fases 4 . En este caso se busca la implementación de las VC en pacientes con ERC que se encuentren en los estadios iniciales de la enfermedad.

\section{Diferencias entre VC y guía de práctica clínica (GPC)}

Tanto las VC como las GPC buscan mejorar la calidad de los procesos y obtener mejores resultados. Sin embargo, las diferencias entre estas dos herramientas se centran en los objetivos que se pretenden alcanzar con las VC al incluir las decisiones organizacionales (quién hace qué, dónde y cuándo) referidas a un contexto estructural o institucional determinado. Según Saturno-Hernández [1], el diseño metodológico de las GPC permite identificar al paciente correcto (diagnóstico) y darle un manejo correcto (tratamiento). Sin embargo, las VC complementan a las GPC dado que su diseño metodológico permite "tratar al paciente correcto con el tratamiento correcto por el profesional o equipo adecuado en el tiempo correcto y en el lugar adecuado en un contexto concreto" [1]; esto último involucrando a toda la institución prestadora de servicios de salud. En Colombia, el Ministerio de Salud y Protección Social y el Instituto de Evaluación Tecnológica en Salud adoptaron la Guía de Práctica Clínica para el diagnóstico y tratamiento de la Enfermedad Renal Crónica para manejar los casos de pacientes con diagnóstico o sospecha de ERC [6]. 


\section{Terminología}

El término más utilizado en inglés para referirse a las VC es Integrated Care Pathways; otros términos son clinical patway, critical patway y Integrated Care Patway, este último usado principalmente en Reino Unido [7]. En español, la expresión más utilizada es "vía clínica” y fue la utilizada en esta investigación.

\section{Mecanismos y objetivos de las VC para lograr una atención clínica de excelencia}

La tabla 1 describe los mecanismos y los objetivos de las VC, así como los resultados esperados al implementarlas para lograr una atención clínica de excelencia en salud renal.

Tabla 1. Mecanismos y objetivos de las vías clínicas para lograr una atención clínica de excelencia en salud renal

\begin{tabular}{|c|c|c|}
\hline Mecanismos & Objetivos & Resultados \\
\hline $\begin{array}{l}\text { * Facilitar la aplicación de las } \\
\text { guías de práctica clínica } \\
\text { * Facilitar la evaluación y la } \\
\text { mejora continua y sistemática } \\
\text { de la atención clínica } \\
\text { * Mejorar la comunicación entre } \\
\text { profesionales y niveles de } \\
\text { atención (lo que es } \\
\text { especialmente útil en el } \\
\text { tratamiento de la enfermedad } \\
\text { renal crónica en los diferentes } \\
\text { estadios de la enfermedad y } \\
\text { niveles de atención) } \\
\text { * Mejorar la comunicación entre } \\
\text { los profesionales y los } \\
\text { pacientes }\end{array}$ & $\begin{array}{l}\text { * Identificar al paciente adecuado } \\
\text { * Proporcionar el tratamiento y } \\
\text { los cuidados adecuados en la } \\
\text { secuencia adecuada en el tiempo } \\
\text { adecuado y en el lugar adecuado, } \\
\text { por parte del personal adecuado, en } \\
\text { un contexto determinado }\end{array}$ & $\begin{array}{l}\text { * Disminuir la variabilidad } \\
\text { no justificada en la } \\
\text { atención clínica } \\
{ }^{*} \text { Alcanzar o sobrepasar } \\
\text { los estándares de calidad } \\
\text { existentes maximizando la } \\
\text { efectividad, la eficiencia, la } \\
\text { seguridad y la satisfacción } \\
\text { de la atención clínica a } \\
\text { través de la atención } \\
\text { centrada en el paciente }\end{array}$ \\
\hline
\end{tabular}

Fuente: elaboración con base en Saturno-Hernández [1].

\section{Experiencias específicas de países con VC en contextos de ERC}

La rápida diseminación de las VC en Estados Unidos explica que más del $60 \%$ de los hospitales de ese país las usen para sus procesos más frecuentes [8]. La primera experiencia de desarrollo e implementación de una VC la llevaron a cabo Zander et al. [9] en 1985 en el New England Medical Center (Boston, Estados unidos); a partir de entonces, estos procesos 
se realizan cada vez con mayor frecuencia y el número de países que han implementado estas herramientas se ha incrementado de forma notable. Los países pioneros en el desarrollo de VC son Reino Unido, Canadá e Irlanda [1].

Es importante destacar que el enfoque de planificar la calidad de la atención sanitaria mediante VC es potencialmente aplicable a cualquier tipo de paciente y proceso asistencial, incluyendo la ERC $[10,11]$.

En Reino Unido se destacan las VC desarrolladas por el National Institute for Health and Care Excellence, en las cuales se compilan condiciones clínicas renales como daño agudo renal, enfermedad renal poliquística autosómica dominante, ERC, enfermedad glomerular y cáncer renal. Estas VC son interactivas y han sido diseñadas para ser usadas en línea; además incluyen diagramas de flujo que permiten acceder a recomendaciones basadas en la evidencia [10].

En Canadá, la VC de la ERC es un recurso dirigido a proveedores de atención primaria que ayuda en el diagnóstico, el manejo médico y la derivación de adultos con esta patología. Este documento contiene recomendaciones basadas en la evidencia de la Kidney Disease Improving Global Outcomes, la Canadian Cardiovascular Society, la Canadian Diabetes Association, la Canadian Hypertension Education Program y la Canadian Society of Nephrology [12].

Navarro-Bermúdez [13] describe el proceso de desarrollo e implementación de una VC en el Hospital de Cádiz (España), el cual atiende cada año a cerca de 300.000 individuos en todos los servicios y a más de 200 en su unidad de hemodiálisis.

En Italia, Genovese et al. [14] demostraron la efectividad del desarrollo y aplicación de una VC al describir un descenso de 11,9\% de las remisiones de pacientes con estadio 5 de ERC a otros centros de atención del país.

El desarrollo de VC en países de Latinoamérica es aún muy incipiente; en Colombia, por ejemplo, solo hay una VC desarrollada por el Ministerio de Salud y Protección Social [15] para el tratamiento de la hepatitis C crónica. Este panorama evidencia la necesidad de crear una cultura de mejora de la calidad a través del desarrollo e implementación de VC, las cuales se complementan con las GPC basadas en la evidencia. 


\section{Conclusiones}

El desarrollo y la implementación de las VC ofrecen grandes oportunidades para la mejora de la calidad de la atención en instituciones prestadoras de servicios de salud, además son de gran utilidad para que clínicos, investigadores y gestores de servicios de salud desarrollen sus labores y para que los pacientes reciban el mejor manejo.

Los clínicos pueden mejorar sus intervenciones a partir de la estandarización de los cuidados que se logran mediante la implementación de las GPC y las VC; esto, además, permite establecer un clima de sinergia multidisciplinaria que genera beneficios tanto para ellos como para los pacientes [8].

Mediante la implementación de la VC, los pacientes y la sociedad en general, verdaderos protagonistas del sistema sanitario, pueden participar en la planificación de sus cuidados a partir de una nueva cultura de empoderamiento que le da un valor añadido a la asistencia actual del sistema general de seguridad social en salud colombiano $[1,2,16,17]$.

Finalmente, se establece que las VC desarrolladas específicamente para ERC podrían ser útiles en las siguientes situaciones:

- Reducción del número de pacientes urémicos que requieren de manera urgente tratamiento de hemodiálisis sin acceso vascular (evitando la inserción de catéteres venosos centrales, los cuales están vinculados a una alta morbilidad en términos de infección y trombosis de las venas centrales y por tanto comprometen la viabilidad de futuros accesos vasculares para hemodiálisis) [3,4,18-20].

- Vigilancia clínica de accesos vasculares para el diagnóstico precoz de disfunción en la sala de diálisis con la ayuda de herramientas diagnósticas como ecografía Doppler color y angiografía [3].

- Manejo clínico y manejo agudo del dolor en adultos con ERC [21-30] y pacientes hemodializados [31,32].

- Mejora de la calidad de la atención a pacientes con ERC en atención primaria en salud $1[2,22,30,33-37]$.

- Manejo clínico de niños con ERC [38].

- Manejo nutricional de pacientes con ERC [31].

- Manejo de dislipidemias en pacientes con trasplante de riñón [39]. 
- Manejo de pacientes con ERC y diabetes [40-43].

- Manejo de pacientes con ERC y falla cardiaca crónica [40].

- Manejo de pacientes embarazadas con ERC $[44,45]$.

\section{Consideraciones éticas}

Los autores declaran que los procedimientos seguidos se realizaron conforme a las normas éticas del comité de experimentación humana responsable y de acuerdo con lo establecido por la Asociación Médica Mundial en la Declaración de Helsinki; que han seguido los protocolos de su centro de trabajo sobre la publicación de datos de pacientes, y que han obtenido el consentimiento informado de los pacientes y/o sujetos referidos en el artículo.

\section{Contribución de los autores}

La conceptualización del estudio estuvo a cargo de Eduardo Navarro Jiménez, Carmen Laborde Cárdenas y Gustavo Aroca Martínez; del desarrollo metodológico se encargaron Eduardo

Navarro Jiménez y Carmen Laborde Cárdenas; la investigación la hicieron Eduardo Navarro Jiménez, Victoria Nicholls De La Hoz, Bryan Olivero Pacheco y Camila Hernández Berrios, y en la redacción (preparación, revisión y edición) del manuscrito participaron todos los autores.

\section{Conflicto de intereses}

Ninguno declarado por los autores.

\section{Financiación}

Este estudio fue patrocinado por la Universidad Simón Bolívar de Barranquilla y la Clínica de la Costa de Barranquilla.

\section{Referencias}

[1] Saturno-Hernández PJ. Cómo lograr la excelencia en la atención sanitaria. Construcción, implementación y evaluación de Vías Clínicas. Cuernavaca: Instituto Nacional de Salud Pública; 2018. $\uparrow$ Ver página 2, 3, 4, 5, 6

[2] Saturno Hernández P. Métodos y herramientas para la realización de ciclos de mejora de la calidad en servicios de salud. Cuernavaca: Instituto Nacional de Salud Pública; 2015. $\uparrow$ Ver página 2, 6 
[3] Ibeas J, Roca-Teyb R, Vallespínc J, Morenod T, Moñuxe G, Martí-Monrós A, et al. Guía Clínica Española del Acceso Vascular para Hemodiálisis. Nefrología. 2017;37(Suppl 1):1-192.

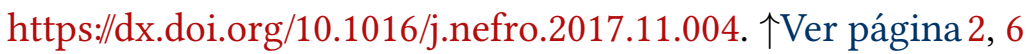

[4] Sou V, McManus C, Mifflin N, Frost SA, Ale J, Alexandrou E. A clinical pathway for the management of difficult venous access. BMC Nurs. 2017;16:64. https://x.doi.org/10.1186/ s12912-017-0261-z. ^Ver página 2, 6

[5] Padilla-Galindo H, Serrano A, Polo A, García R, Acosta J, Navarro-Micr E, et al. Caracterización clínica-epidemiológica de las glomerulonefritis primarias un centro de referencia de caribe colombiano, en niños menores de 15 años, de enero 2008 a diciembre 2013. Rev. Colomb. Nefrol. 2014;1(Suppl 1). $\uparrow$ Ver página 2

[6] Colombia. Ministerio de Salud y Protección Social (MinSalud). Guía de Práctica Clínica para el diagnóstico y tratamiento de la Enfermedad Renal Crónica (adopción). Bogotá D.C.: MinSalud; 2016 [citado mayo 19 2021]. Disponible en: http:/gpc.minsalud.gov.co/gpc_sites/ Repositorio/Otros_conv/GPC_e_renal/gpc_e_renal.aspx. $\uparrow$ Ver página 3

[7] Every NR, Hochman J, Becker R, Kopecky S, Cannon CP. Critical Pathways: a review. Committee on Acute Cardiac Care, Council on Clinical Cardiology, American Heart Association. Circulation. 2000;101(4):461-5. https://dx.doi.org/10.1161/01.cir.101.4.461. $\uparrow$ Ver página 4

[8] Carrasco G, Ferrer J. Las vías clínicas basadas en la evidencia como estrategia para la mejora de la calidad: metodología, ventajas y limitaciones. Rev. Calid. Asist. 2001;16(3):199-207. https://dx.doi.org/10.1016/S1134-282X(01)77405-2. 个Ver página 4, 6

[9] Zander KS, Bower KA, Etheredge MLS. Nursing Case Management: Blueprints for Transformation. Boston: New England Medical Center Hospitals; 1987. $\uparrow$ Ver página 4

[10] The National Institute for Health and Care Excellence (NICE). Kidney conditions overview. Londres: NICE; 2018 [citado mayo 24 2021]. Disponible en: https:/pathways.nice.org.uk/pathways/kidney-conditions\#content=view-node\% 3Anodes-autosomal-dominant-polycystic-kidney-disease. $\uparrow$ Ver página 5

[11] University of Calgary. Chronic Kidney Disease Clinical Pathway. Calgary: University of

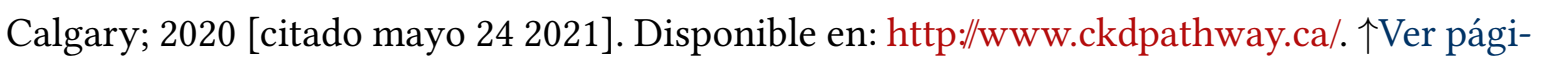
na 5

[12] Interdisciplinary Chronic Disease Collaboration \& Southern Alberta Renal Program. Chronic Kidney Disease (CKD) Clinical Pathway. 2020 [citado mayo 24 2021]. Disponible en: http:/www.ckdpathway.ca/aboutthepathway. $\uparrow$ Ver página 5 
[13] Navarro-Bermúdez M. Desarrollo e implementación de una vía clínica a los pacientes que inician la hemodiálisis de forma programada. Rev Soc Esp Enferm Nefrol. 2011;14(1):15-22. 个Ver página 5

[14] Genovese C, DE Belvis AG, Rinaldi M, Manno V, Squeri R, LA Fauci V, et al. Quality and management care improvement of patients with chronic kidney disease: from data analysis to the definition of a targeted clinical pathway in an Italian Region. J Prev Med Hyg.

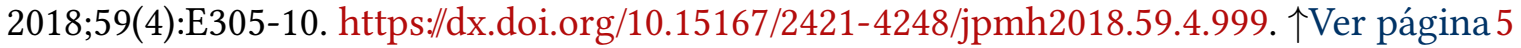

[15] Colombia. Ministerio de Salud y Protección Social (MinSalud). Vía clínica para el tratamiento de hepatitis C crónica. Bogotá D.C.: MinSalud; 2017 [citado mayo 13 2021]. Disponible en: https:/www.minsalud.gov.co/sites/rid/Lists/BibliotecaDigital/RIDE/VS/MET/ via-clinica-tratamiento-hepatitisc.pdf. $\uparrow$ Ver página 5

[16] Jimenez N, Aroca-Martínez G, Santos GD. Implementación de un modelo de salud renal en red informática para la temprana detección y cuidado de la nefropatía primaria lúpica y glomerulonefritis en la Región Caribe Colombiana. Rev. Colomb. Nefrol. 2014;1(Suppl 1). 个Ver página 6

[17] Castillo-Parodi L, Navarro-Jiménez E, Arango-Quiroz Y, López-Avendaño A, Mejía-Varela V, González-Torres HJ, et al. Obesity Association with Chronic Renal Disease in Patients attended at Clínica de la Costa. Barranquilla, Colombia. 2005-2014. Rev. Colomb. Nefrol. 2021;3(1):14-9. https://doi.org/10.22265/acnef.3.1.217. 个Ver página 6

[18] Genovese C, DE Belvis AG, Rinaldi M, Manno V, Squeri R, LA Fauci V, et al. Quality and management care improvement of patients with chronic kidney disease: from data analysis to the definition of a targeted clinical pathway in an Italian Region. J Prev Med Hyg.

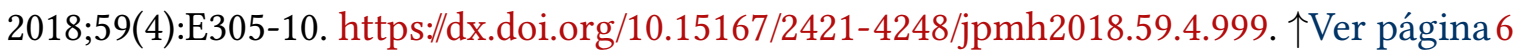

[19] Childrens Hospital Colorado. Venous thromboembolism (VTE) prevention. Clinical pathway; 2020 [citado mayo 24 de 2021]. Disponible en: https:/www. childrenscolorado.org/4adc94/globalassets/healthcare-professionals/clinical-pathways/ venous-thromboembolism.pdf. $\uparrow$ Ver página 6

[20] Canadá. Ministry of Health and Long-Term Care. Quality-Based Procedures Clinical Handbook for Chronic Kidney Disease Ontario: Ministry of Health and Long-Term Care; 2016 [citado mayo 24 2021]. Disponible en: http:/www.health.gov.on.ca/en/pro/programs/ ecfa/docs/qbp_kidney.pdf. $\uparrow$ Ver página 6

[21] Curtis C, Balint C, Al Hamarneh YN, Donald M, Tsuyuki RT, McBrien K, et al. Online clinical pathway for managing adults with chronic kidney disease. Can Pharm J (Ott).

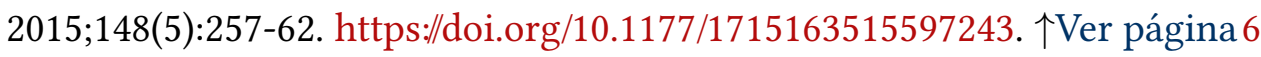


[22] Donald M, McBrien K, Jackson W, Manns BJ, Tonelli M, King-Shier K, et al. Development and implementation of an online clinical pathway for adult chronic kidney disease in primary care: a mixed methods study. BMC Med Inform Decis Mak. 2016;16:109. https://doi.org/10.1186/s12911-016-0350-z. 个Ver página 6

[23] Gruber-Baldini AL, Velozo C, Romero S, Shulman LM. Validation of the PROMIS® measures of self-efficacy for managing chronic conditions. Qual. Life Res. 2017;26(7):1915-24. https://doi.org/10.1007/s11136-017-1527-3. 个Ver página 6

[24] Johnson ML, Zimmerman L, Welch JL, Hertzog M, Pozehl B, Plumb T. Patient activation with knowledge, self-management and confidence in chronic kidney disease. J Ren Care.

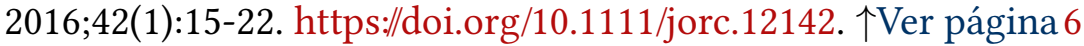

[25] Ploeg J, Matthew-Maich N, Fraser K, Dufour S, McAiney C, Kaasalainen S, et al. Managing multiple chronic conditions in the community: a Canadianqualitative study of the experiences of older adults, family caregivers and healthcare providers. BMC Geriatr. 2017;17(1):40. https://oi.org/10.1186/s12877-017-0431-6. 个Ver página 6

[26] Dobkowski D, Zuber K, Davis J. Updated guidelines for managing chronic kidney disease. JAAPA. 2013;26(11):27-31. https://doi.org/10.1097/01.JAA.0000436686.98552.56. 个Ver página 6

[27] Post FA. Managing chronic kidney disease in the older adults living with HIV. Curr Opin Infect Dis. 2017;30(1):4-11. https://doi.org/10.1097/QCO.0000000000000333. $\uparrow$ Ver página 6

[28] Coyne E, Langham H, Tomlin M, Hope W, Johnson C, Byrne C, et al. Young adults with chronic kidney disease: An exploration of their relationships and support networks. J Ren Care. 2019;45(1):20-8. https://doi.org/10.1111/jorc.12263. $\uparrow$ Ver página 6

[29] Toy JM, Drechsler A, Waters RC. Clinical pathways for primary care: current use, interest and perceived usability. J Am Med Inform Assoc. 2018;25(7):901-6. https://doi.org/10.1093/ jamia/ocy010. $\uparrow$ Ver página 6

[30] Grimsmo A, Løhre A, Røsstad T, Gjerde I, Heiberg I, Steinsbekk A. Disease- specific clinical pathways - are they feasible in primary care? A mixed-methods study. Scand J Prim

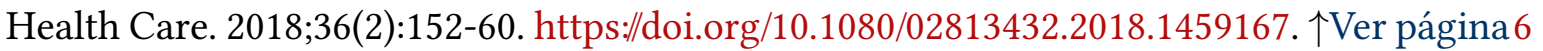

[31] Mejia C, Libby BA, Bracken ML, Shanley B, Holovatska MM, Wanik J, et al. Interest in Digital Dietary Support Among Adults With Kidney Failure Receiving Hemodialysis. J Ren Nutr. 2020;31(3):327-32. https://oi.org/10.1053/j.jrn.2020.06.004. $\uparrow$ Ver página 6 
[32] Harwood L, Clark AM. Dialysis modality decision-making for older adults with chronic kidney disease. J Clin Nurs. 2014;23(23-24):3378-90. https:/doi.org/10.1111/jocn.12582. $\uparrow$ Ver página 6

[33] Tam-Tham H, Hemmelgarn BR, Campbell DJ, Thomas CM, Fruetel K, Quinn RR, et al. Primary care physicians' perceived barriers, facilitators and strategies to enhance conservative care for older adults with chronic kidney disease: a qualitative descriptive study. Nephrol Dial Transplant. 2016;31(11):1864-70. https://doi.org/10.1093/ndt/gfv408. 个Ver página 6

[34] Tahir M, Hassan S, de Lusignan S, Shaheen L, Chan T, Dmitrieva O. Development of a questionnaire to evaluate practitioners' confidence and knowledge in primary care in managing chronic kidney disease. BMC Nephrol. 2014;15:73. https:/doi.org/10.1186/ 1471-2369-15-73. $\uparrow$ Ver página 6

[35] Owen JE, Walker RJ, Edgell L, Collie J, Douglas L, Hewitson TD, et al. Implementation of a pre-dialysis clinical pathway for patients with chronic kidney disease. Int J Qual Heal Care. 2006;18(2):145-51. https://doi.org/10.1093/intqhc/mzi094. $\uparrow$ Ver página 6

[36] Tsuyuki RT. Reaffirming our focus on patient-centred care. Can Pharm J (Ott).

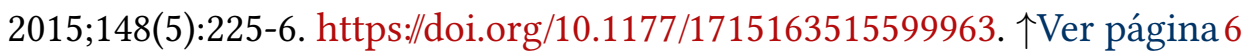

[37] Smekal MD, Tam-Tham H, Finlay J, Donald M, Benterud E, Thomas C, et al. Perceived Benefits and Challenges of a Risk-Based Approach to Multidisciplinary Chronic Kidney Disease Care: A Qualitative Descriptive Study. Can J Kidney Health Dis. 2018;5:205435811876380.

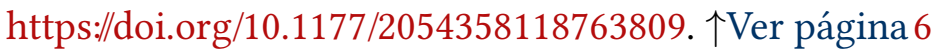

[38] Geense WW, van Gaal BG, Knoll JL, Cornelissen EA, Schoonhoven L, Kok G. Online Support Program for Parents of Children With a Chronic Kidney Disease Using Intervention Mapping: A Development and Evaluation Protocol. JMIR Res. Protoc. 2016;5(1):e1. https:/ doi.org/10.2196/resprot.4837. $\uparrow$ Ver página 6

[39] Kasiske B, Cosio FG, Beto J, Bolton K, Chavers BM, Grimm R Jr, et al. Clinical practice guidelines for managing dyslipidemias in kidney transplant patients: a report from the Managing Dyslipidemias in Chronic Kidney Disease Work Group of the National Kidney Foundation Kidney Disease Outcomes Quality Initiative. Am J Transplant. 2004;4(Suppl 7):13-53.

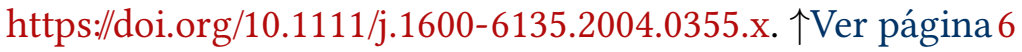

[40] Lovre D., Shah S, Sihota A, Fonseca VA. Managing Diabetes and Cardiovascular Risk in Chronic Kidney Disease Patients. Endocrinol Metab Clin North Am. 2018;47(1):237-57.

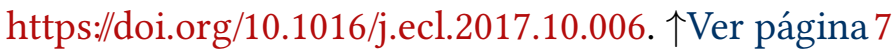

[41] Zac-Varghese S, Winocour P. Managing diabetic kidney disease. Br Med Bull. 2018;125(1):55-66. https:/doi.org/10.1093/bmb/ldx047. 个Ver página 7 
[42] Clemens KK, O’Regan N, Rhee JJ. Diabetes Management in Older Adults With Chronic Kidney Disease. Curr Diab Rep. 2019;19(3):11. https://doi.org/10.1007/s11892-019-1128-3. 个Ver página 7

[43] Lo C, Zimbudzi E, Teede H, Cass A, Fulcher G, Gallagher M, et al. Models of care for comorbid diabetes and chronic kidney disease. Nephrology (Carlton). 2018;23(8):711-7. https: //doi.org/10.1111/nep.13232. 个Ver página 7

[44] Fitzpatrick A, Mohammadi F, Jesudason S. Managing pregnancy in chronic kidney disease: improving outcomes for mother and baby. Int J Womens Health. 2016;8:273-85. https://doi.org/10.2147/IJWH.S76819. 个Ver página 7

[45] Wiles K, Chappell L, Clark K, Elman L, Hall M, Lightstone L, et al. Clinical practice guideline on pregnancy and renal disease. BMC Nephrol. 2019;20(1):401. https://doi.org/10.1186/ s12882-019-1560-2. $\uparrow$ Ver página 7 